\title{
Segmentation of large renal tumors in CT images by the integration of deep neural networks and thresholding
}

\author{
Nasim Nasiri, Saeed Mohagheghi, and Amir Hossein Foruzan* \\ Department of Biomedical Engineering, Engineering Faculty, Shahed University, Tehran, Iran. \\ nasim.nasirieshahed.ac.ir, s.mohagheghi@shahed.ac.ir, \\ "a.foruzaneshahed.ac.ir (Corresponding author).
}

\begin{abstract}
To segment the kidney and its large tumors, we combine a deep neural network and thresholding technique. The deep network segments kidney, and its output is used to detect probable renal tumors. We compare the kidney volume with a normal kidney shape. Incomplete shapes are searched for tumors. Using a seed point the center of the tumor cluster is defined. Then, the pixels of a slice is labeled as normal or abnormal. The labeled pixels are post-processed using morphological filters to refine the result. The outcome of the algorithm is the tumor volume.
\end{abstract}

\section{Introduction}

The texture of the kidney includes two separate parts: cortex and pyramid. A renal tumor exists either as a small region completely inside the kidney or a large mass that deforms the shape of the organ as well. Regarding its texture, there are both homogeneous or inhomogeneous tumors. The special character of a renal tumor is its compactness and its nearly spherical shape.

Among various segmentation technique, Deep Neural Network (DNN) is considered as a recent tool that has attracted many researchers. However, it is not matured and the research to develop new variations continue.

To segment the kidney, we use a DNN and give its output to a thresholding clustering algorithm to delineate the boundary of renal tumors. When the tumor is of the large type, it has an irregular shape texture and a DNN fails to segment its boundary correctly. Therefore, the shape of the segmented organ is different from a normal organ. We use this feature to detect a probable abnormality and to delineate its boundary. As the innovation of the proposed method, we use the segmented kidney volume to detect abnormalities and utilize the thresholding technique to segment large renal tumors.

\section{The Proposed Method}

The flowchart of our method is shown in Fig. 1. It consists of kidney segmentation and tumor detection and delineation steps. After loading CT data, the necessary pre- 
processing are applied to it. Then, a DNN performs kidney segmentation. The output of the DNN is analyzed to detect any probable tumors that are segmented by the Kmeans algorithm in the next step.

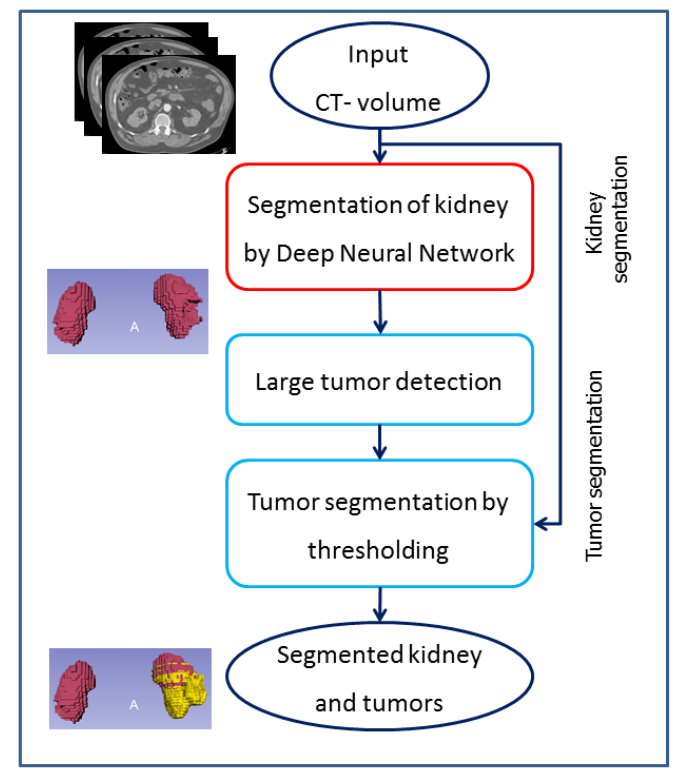

Fig. 1- The flowchart of the proposed method.

\subsection{Kidney Segmentation with DNN}

All dataset were pre-processed, by applying a recursive Gaussian smoothing, intensity windowing (between 0 and 500) and resampling to $128 \times 128 \times 128$ voxels. Our kidney segmentation model is an improved version of 3D UNet [1], which is a Fully Convolutional Network (FCN) [2] with seven convolutional (Conv) steps in each of the analysis and synthesis paths. For each step, we design a Conv block containing a $3 \mathrm{D}$ Conv layer with a $3 \times 3 \times 3$ kernel size followed by a batch normalization $(\mathrm{BN})$ layer and an Exponential Rectified Linear unit (ELu) [3]. At the final layer, a Conv layer with sigmoid activation function predicts the kidney probability map, which is thresholded to produce the final binary mask (Fig. 2). The Dice coefficient is used as the loss function.

To improve the performance of the network, we augmented the dataset by rotating and flipping images, and used 90 percent of the shuffled dataset as train and 10 percent as the validation images. The TensorFlow [4] framework is used to implement the model, and the optimization process performed for 200 epochs on a GeForce ${ }^{\circledR}$ GTX $^{\text {TM }} 1070$ Ti graphics card with 8GB dedicated memory. 
Fig. 2- The architecture of the kidney segmentation DNN.

\subsection{Abnormality Detection}

Concerning large renal tumors, it deforms the shape of the organ. The output of the DNN ignores tumors and output an incomplete kidney shape. We detect the defected shape by comparing it to a normal kidney. We use an affine registration scheme to align a normal kidney shape to the output of the DNN. We put the center-of-mass of the normal organ on the other shape and use the Principal Component Analysis (PCA) to extract the direction of the point distributions. Three vectors are obtained corresponding to a shape. The three vectors are aligned together and the normal shape is scaled to fit the size of the segmented kidney. Details of the registration scheme are given in [5]. Then, the symmetric Hausdorff distance is measured and it is used as an index of completeness. In Fig. 3, the typical incomplete shape of the kidney corresponding to data\#215 is shown. A large index indicates a defect on the output of the DNN. At this step, we treat complete shapes as normal and we plan to consider segmentation of small tumors in the future.

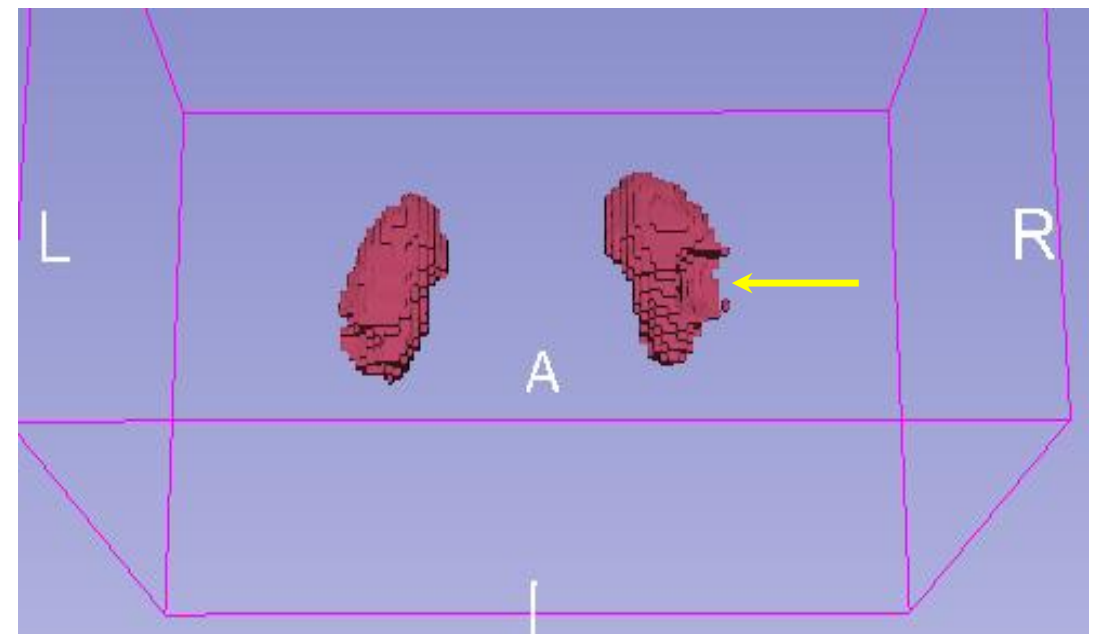

Fig. 3- Incomplete shape of the kidney corresponding to data\#215 is shown. The incomplete volume is shown by an arrow (corresponding to data \#215).

\subsection{Tumor Segmentation}

We use an incomplete kidney mask and dilate it by a morphological filter with a disk structuring element of size 3 to estimate the location of the tumor. The dilation of the mask is performed restricted to the region of the normal shape. The distance of the points inside the dilated region is calculated and a typical far point is selected as a seed. Then, 2D CT slices are processed one-by-one to label abnormal pixels. The intensity of a $3 \times 3$ neighborhood of the seed point is used as the feature vector of the tumor region. The average of $5 \times 5$ pixels around the seed point is utilized as the center 
of the tumor cluster to compensate for noise and texture inhomogeneity. The distance of all pixels in a slice to the center of the cluster is calculated and those with a distance less than 90 are labeled as abnormal points. The threshold is obtained using the validation set and then it is fixed for all test data to follow the rules of the challenge.

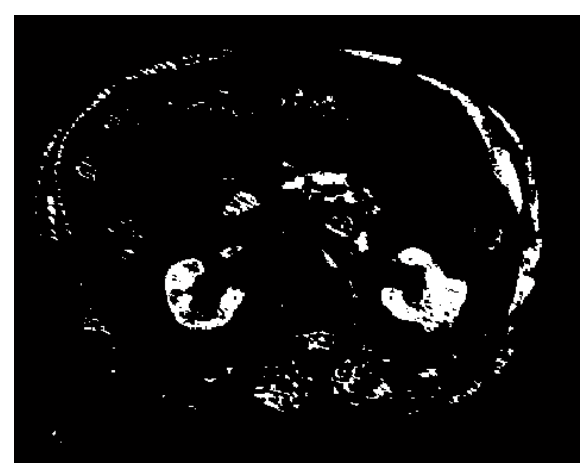

(a)

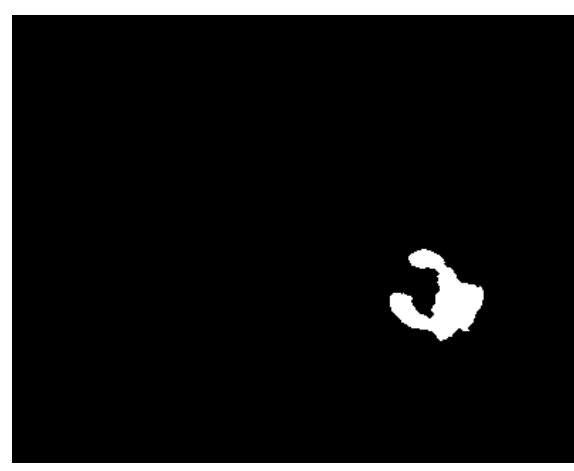

(b)

Fig. 4- (a) Thresholding labels non-kidney pixels as a tumor. (b) Post-processing of the results remains only kidney and tumor (corresponding to data \#215).

Since the texture of a tumor is similar to those of the tissues in the abdominal region, the output of the above step contains non-kidney pixels as well. We post-process the output of the thresholding by removing objects smaller than 50 pixels, remaining the object having an intersection with the seed point, morphological closing filtering, and morphological hole filling (Fig. 4). The output object is considered as the tumor and its center-of-mass replaces the current seed point and it is used in the next slice. Based on our experience, we do not update the center of the tumor cluster since it leads to over-segmentation. Slices are processed one after the other until no pixel is labeled as abnormal. Concatenation of 2D slices makes the tumor volume (Fig. 5).

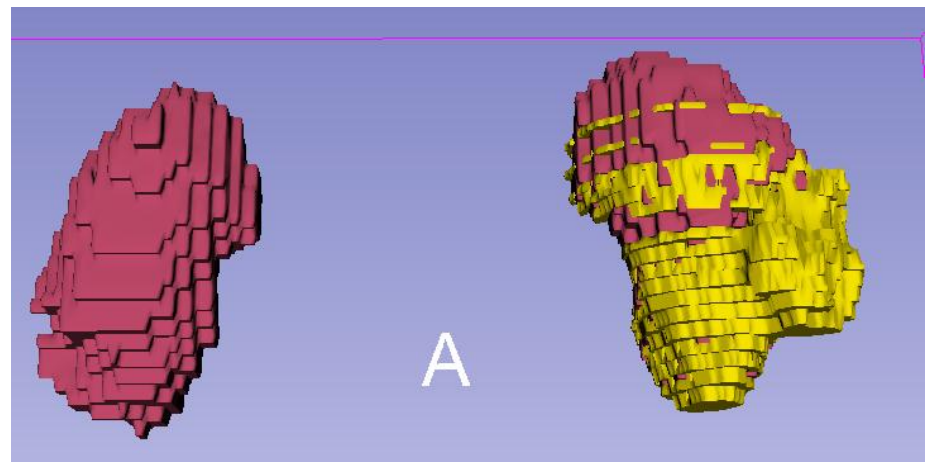

Fig. 5- The volume of the segmented kidney (brown) and its tumor (yellow) are shown (corresponding to data \#215). 
The proposed algorithm was implemented in MATLAB 2014 environment and visualizations were performed using the 3D-Slicer software [6].

\section{Discussion, Conclusion and Future Works}

Incomplete kidney region is an important sign of a large tumor that we employed it for abnormality segmentation. When there is only one kidney in the abdominal region, the DNN cannot ignore it. There were some cases that our tumor segmentation algorithm failed as well. However, our method enhances the output of the deep network.

As improvements of the proposed method, we plan to use a more complex feature vector to model inhomogeneous texture more accurately. Inclusion of geometric constraints prevents over-segmentation as well. Since we had to follow the rules of the challenge, we did not use interactions. In the future, we decide to segment cortex and pyramid parts of a kidney to search for small tumors as well.

\section{References}

1. Çiçek Ö, Abdulkadir A, Lienkamp SS, Brox T, Ronneberger O (2016) 3D U-Net: learning dense volumetric segmentation from sparse annotation. In: International Conference on Medical Image Computing and Computer-Assisted Intervention. Springer, pp 424-432.

2. Long J, Shelhamer E, Darrell T (2015) Fully convolutional networks for semantic segmentation. In: Proceedings of the IEEE conference on computer vision and pattern recognition. pp 3431-3440.

3. Clevert D-A, Unterthiner T, Hochreiter S (2015) Fast and accurate deep network learning by exponential linear units (elus). arXiv Prepr arXiv151107289.

4. Abadi M, Barham P, Chen J, Chen Z, Davis A, Dean J, Devin M, Ghemawat S, Irving G, Isard M (2016) Tensorflow: a system for large-scale machine learning. In: OSDI. pp 265-283.

5. Foruzan, AH, and Rajabzadeh Motlagh H. "Multimodality liver registration of Open-MR and CT scans." International journal of computer assisted radiology and surgery 10, no. 8 (2015): 1253-1267.6. Slicer, http://www.Slicer.org/. Accessed: 10 July., 2019. 